\title{
Long intergenic non-coding RNA LINC01232 contributes to esophageal squamous cell carcinoma progression by sequestering microRNA-654-3p and consequently promoting hepatoma-derived growth factor expression
}

\author{
MEIHUA ZHAO $^{1}$, HAISHAN CUI ${ }^{2}$, BAISUI ZHAO ${ }^{1}$, MEI LI $^{1}$ and HAIQING MAN ${ }^{2}$ \\ Departments of ${ }^{1}$ Gastroenterology and ${ }^{2}$ Endoscopy, Affiliated Hospital of Inner Mongolia University for The Nationalities, \\ Tongliao, Inner Mongolia 028007, P.R. China
}

Received June 4, 2020; Accepted August 20, 2020

DOI: $10.3892 / \mathrm{ijmm} .2020 .4750$

\begin{abstract}
Long intergenic non-coding RNA 01232 (LINC01232) was identified as a critical regulator of the development of pancreatic adenocarcinoma. The present study investigated the expression and regulatory roles of LINC01232 in esophageal squamous cell carcinoma (ESCC). The main aim of the present study was to elucidate the underlying mechanisms through which LINC01232 affects the malignancy of ESCC. Initially, LINC01232 expression in ESCC was analyzed using the TCGA and GTEx databases and was confirmed using reverse transcription-quantitative polymerase chain reaction. ESCC cell proliferation, apoptosis and migration and invasion were assessed using the Cell Counting kit- 8 assay, flow cytometric analysis, and migration and invasion assays, respectively. ESCC tumor growth in vivo was examined using a xenograft mouse model. As shown by the results, a high LINC01232 expression was detected in ESCC tissues and cell lines. LINC01232 downregulation suppressed the proliferation, migration and invasion of ESCC cells, and promoted cell apoptosis in vitro. In addition, LINC01232 depletion restricted tumor growth in vivo. Mechanistically, LINC01232 was shown to function as an microRNA-654-3p (miR-654-3p) sponge in ESCC cells, and hepatoma-derived growth factor (HDGF) was identified as a direct target of miR-654-3p. LINC01232 could bind competitively to miR-654-3p and decrease its expression in ESCC cells, thereby promoting HDGF expression. Rescue experiments reconfirmed that the effects of LINC01232 deficiency in ESCC cells were restored by increasing the output of the miR-654-3p/HDGF axis. On the whole, the present
\end{abstract}

Correspondence to: Professor Meihua Zhao, Department of Gastroenterology, Affiliated Hospital of Inner Mongolia University for The Nationalities, 1742 East Huolinhe Street, Tongliao, Inner Mongolia 028007, P.R. China

E-mail: zhaomeihua_inner@126.com

Key words: hepatoma-derived growth factor, esophageal squamous cell carcinoma, theoretical basis, competing endogenous RNA study demonstrates that LINC01232 plays a tumor-promoting role during the progression of ESCC by regulating the miR-654-3p/HDGF axis. The LINC01232/miR-654-3p/HDGF pathway may thus provide a novel theoretical basis for the management of ESCC.

\section{Introduction}

Esophageal cancer is the seventh most common type of human cancer and the 6th leading cause of cancer-associated mortality worldwide (1). More than 455,800 newly diagnosed esophageal cancer cases and 400,200 related deaths are reported worldwide each year (2), and the majority of these cases are detected in Asia, Northern France, and Eastern and Southern Africa (3). Esophageal squamous cell carcinoma (ESCC), the major histological type of esophageal cancer, accounts for approximately $90 \%$ of all esophageal cancer cases (4). Approximately half of all patients with ESCC present with advanced-stage disease at the time of the initial diagnosis, and these patients thus have poor therapeutic responses following surgical resection (5). Despite tremendous advances being made in multidisciplinary therapies involving surgical excision and chemoradiotherapy, the clinical efficacy of treatment for ESCC remains largely unfavorable, and the 5-year survival rate is $<25 \%$. Multiple factors, including the excessive consumption of alcohol, smoking, the consumption of very hot tea and red meat, and a low intake of fruits and vegetables, have been identified to be closely associated with the pathogenesis of ESCC (6,7); however, the complex mechanistic events remain largely unknown. Hence, the determination of the detailed mechanisms closely associated with the pathogenesis of ESCC would represent a highly significant step toward improving the diagnostic techniques and therapeutics for ESCC.

Long non-coding RNAs (lncRNAs) are a class of long nucleotide transcripts ( $>200$ nucleotides in length) that are incapable of coding proteins (8). IncRNAs contribute to the control of gene expression at the transcriptional, post-transcriptional and chromosomal levels (9). A number of studies have verified the regulatory effects of IncRNAs in numerous normal physiological and pathological processes (10-12). In recent decades, the significance of IncRNAs in carcinogenesis 
and progression has been recognized by researchers (13-15). The differential expression of various lncRNAs in ESCC has been identified, and some lncRNAs have been demonstrated to be crucial contributors to the oncogenicity of ESCC via their cancer-inhibiting or cancer-promoting activities (16-18).

MicroRNAs (miRNAs or miRs) are a class of endogenous, highly conserved and non-coding RNA transcripts with lengths of 17-23 nucleotides (19). miRNAs participate in the regulation of gene expression at the post-transcriptional level by binding directly to the 3'-untranslated regions (3'-UTRs) of target genes and consequently triggering the degradation of mRNAs and/or inhibiting translation (20). Research has highlighted the necessary role of miRNAs in the onset and progression of ESCC (21). Existing evidence has demonstrated the dysregulation of miRNAs in ESCC and the capacities of these molecules to regulate various malignant phenotypes (22-24). The competing endogenous (ce)RNA hypothesis suggests that lncRNAs function predominantly as molecular sponges to decrease the availability of target miRNAs, thereby reducing the suppressive effects of miRNAs on their downstream mRNA targets (25). Therefore, an exploration of the lncRNAs and miRNAs involved in the oncogenesis and development of ESCC may help identify potential targets for the diagnosis, prevention, and treatment of this malignancy.

Long intergenic non-coding RNA LINC01232 has been identified as a critical regulator of pancreatic adenocarcinoma oncogenesis (26). However, the involvement of LINC01232 in ESCC has not yet been thoroughly elucidated. In the present study, the pattern of LINC01232 expression in ESCC was examined, and the regulatory roles of this lncRNA in ESCC cells, as well as the underlying mechanisms were investigated.

\section{Materials and methods}

Clinical tissues, cell culture and cell transfection. A total of 55 pairs of ESCC tissues and matched normal tissues were collected from patients (34 males, 21 females; age range, 57-74 years) with ESCC at the Affiliated Hospital of Inner Mongolia University for the Nationalities. Follow-up was performed through outpatient visits or via telephone (every 1-2 months). All tissues were obtained from patients who had not undergone chemotherapy or radiotherapy prior to surgical excision. Immediately following excision, all tissue specimens were snap-frozen and stored in liquid nitrogen prior to RNA isolation. Human clinical tissues were collected and used in accordance with the Institutional Ethics Committee of the Affiliated Hospital of Inner Mongolia University for the Nationalities (\#17-0602). Written informed consent was provided by all patients prior to tissue collection.

A total of 3 ESCC cell lines, Eca109, TE-1 and KYSE150, were acquired from the Type Culture Collection of the Chinese Academy of Sciences. The Eca109 and TE-1 cells were grown in RPMI-1640 medium (Gibco; Thermo Fisher Scientific, Inc.), while the KYSE150 cells were cultured in RPMI-1640/F12 medium (Gibco; Thermo Fisher Scientific, Inc.). Both types of basal media were supplemented with $10 \%$ fetal bovine serum (FBS; Gibco; Thermo Fisher Scientific, Inc.) and $1 \%$ penicillin/streptomycin (Gibco; Thermo Fisher Scientific, Inc.). The normal human esophageal epithelial cell line, HET-1A, was obtained from the American Type Culture
Collection (ATCC) and was cultured in BEGM medium (Lonza/Clonetics Corporation) supplemented with 10\% FBS. All cells were cultured at $37^{\circ} \mathrm{C}$ in a humidified incubator containing $5 \% \mathrm{CO}_{2}$.

Small interfering RNAs (siRNAs) specific for LINC01232 (si-LINC01232\#1, si-LINC01232\#2 and si-LINC01232\#3) and scrambled siRNAs (si-ctrl) were generated by Ribobio (Guangzhou RiboBio Co., Ltd.). The si-LINC01232 sequences were as follows: si-LINC01232 \#1, 5'-GTGTAATTTCAC TTGAATAAAT-3'; si-LINC01232 \#2, 5'-GTGCGTTTTGTC CAATTATTTGT-3'; and si-LINC01232 \#3, 5'-GACTTCTTA GAACTAAATTGAAA-3'. The si-NC sequence was 5'-CAC GATAAGACAATGTATTT-3'. miR-654-3p mimic, negative control (NC) miRNA mimic, miR-654-3p inhibitor and NC inhibitor were purchased from GenePharma. The plasmid used to induce HDGF overexpression (pcDNA3.1-HDGF) was produced by GenePharma, and the empty pcDNA3.1 plasmid vector was used as the negative control. Cells were cultured in 6-well plates and transfected with miRNA mimics (100 pmol), miRNA inhibitors (100 pmol), siRNAs (100 pmol), or plasmids $(4 \mu \mathrm{g})$ using Lipofectamine 2000 reagent (Invitrogen; Thermo Fisher Scientific, Inc.). Reverse transcription-quantitative polymerase chain reaction (RT-qPCR), flow cytometric analysis, and migration and invasion assays were conducted at $48 \mathrm{~h}$ post-transfection. Cell Counting kit-8 (CCK-8) assay and western blot analysis were performed following 24 and $72 \mathrm{~h}$ of incubation, respectively.

Isolation of cell cytosolic/nuclear fractions. The nuclear and cytosolic fractions of ESCC cells were separated using the Cytoplasmic \& Nuclear RNA Purification kit (Norgen Biotek Corp.) in accordance with the manufacturer's protocol. RNA was isolated from the nuclear and cytosolic fractions and used in RT-qPCR assays to determine the distribution of LINC01232 in ESCC cells.

$R T$ - $q P C R$. TRIzol reagent (Invitrogen; Thermo Fisher Scientific, Inc.) was used to extract total RNA. To quantify the expression of LINC01232 and HDGF, reverse transcription was conducted using the PrimeScript ${ }^{\mathrm{TM}}$ RT reagent kit with gDNA Eraser (Takara, Dalian, China). The resultant first-strand cDNA was used as a template for qPCR, which was performed using TB Green ${ }^{\circledR}$ Premix Ex Taq ${ }^{\mathrm{TM}}$ II (Takara Biotechnology Co., Ltd.). To analyze the expression of miR-654-3p, the Mir-X miRNA First-Strand Synthesis kit (Takara Biotechnology Co., Ltd.) was used to prepare cDNA, and the Mir-X miRNA qRT-PCR TB Green ${ }^{\circledR}$ Kit was then used to perform qPCR (Takara Biotechnology Co., Ltd.). $G A P D H$ and U6 small nuclear RNA were used as the reference transcripts for lncRNA/mRNA and miRNA, respectively. All data were analyzed using the $2^{-\Delta \Delta \mathrm{Cq}}$ method (27). All primer sequences are listed in Table I.

CCK-8 assay. The CCK-8 assay was conducted to determine cell proliferation. At $48 \mathrm{~h}$ following transfection, cells were collected and seeded into 96-well plates. Each well contained $100 \mu \mathrm{l}$ of cell suspension containing $2 \times 10^{3}$ cells. The cells were incubated in a humidified incubator with $5 \% \mathrm{CO}_{2}$ at $37^{\circ} \mathrm{C}$. At $0,24,48$ and $72 \mathrm{~h}$ following cell inoculation, the culture medium was carefully discarded, and $100 \mu \mathrm{l}$ of fresh 
Table I. Sequences of primers used in RT-qPCR.

\begin{tabular}{|c|c|}
\hline Gene & Sequences $\left(5^{\prime}-3{ }^{\prime}\right)$ \\
\hline \multirow[t]{2}{*}{ LINC01232 } & Forward: AAAACCTTGAAATCCСТTAАTACCA \\
\hline & Reverse: CCTTACCCGTGGAATTCACATATA \\
\hline \multirow[t]{2}{*}{ HDGF } & Forward: AATCAACAGCCAACAAATACCAAGT \\
\hline & Reverse: AGCCTTGACAGTAGGGTTGTTCTC \\
\hline \multirow[t]{2}{*}{ GAPDH } & Forward: CGGAGTCAACGGATTTGGTCGTAT \\
\hline & Reverse: AGCCTTCTCCATGGTGGTGAAGAC \\
\hline \multirow[t]{2}{*}{$\operatorname{miR}-122-5 p$} & Forward: TCGGCAGGUGGAGUGUGACAAUG \\
\hline & Reverse: CACTCAACTGGTGTCGTGGA \\
\hline \multirow[t]{2}{*}{$\operatorname{miR}-181 b-5 p$} & Forward: TCGGCAGGGGUCACAAUCAACAUUC \\
\hline & Reverse: CACTCAACTGGTGTCGTGGA \\
\hline \multirow[t]{2}{*}{$\operatorname{miR}-181 c-5 p$} & Forward: TCGGCAGGGGUUUGGGGGAACAUUC \\
\hline & Reverse: CACTCAACTGGTGTCGTGGA \\
\hline \multirow[t]{2}{*}{ miR-181d-5p } & Forward: TCGGCAGGGGUCACAAUCAACAUUC \\
\hline & Reverse: CACTCAACTGGTGTCGTGGA \\
\hline \multirow[t]{2}{*}{$\operatorname{miR}-211-5 p$} & Forward: TCGGCAGGUUCCCUUUGUCAUCC \\
\hline & Reverse: CACTCAACTGGTGTCGTGGA \\
\hline \multirow[t]{2}{*}{$\operatorname{miR}-370-5 p$} & Forward: TCGGCAGGCAGGUCACGUCUC \\
\hline & Reverse: CACTCAACTGGTGTCGTGGA \\
\hline \multirow[t]{2}{*}{ miR-493-5p } & Forward: TCGGCAGGUUGUACAUGGUAGG \\
\hline & Reverse: CACTCAACTGGTGTCGTGGA \\
\hline \multirow[t]{2}{*}{ miR-506-5p } & Forward: TCGGCAGGUAUUCAGGAAGGUG \\
\hline & Reverse: CACTCAACTGGTGTCGTGGA \\
\hline \multirow[t]{2}{*}{ miR-590-5p } & Forward: TCGGCAGGGAGCUUAUUCAUAA \\
\hline & Reverse: CACTCAACTGGTGTCGTGGA \\
\hline \multirow[t]{2}{*}{ miR-642b-5p } & Forward: TCGGCAGGGGUUCCCUCUCCA \\
\hline & Reverse: CACTCAACTGGTGTCGTGGA \\
\hline \multirow[t]{2}{*}{$\operatorname{miR}-654-3 p$} & Forward: TCGGCAGGUGGUGGGCCGCAG \\
\hline & Reverse: CACTCAACTGGTGTCGTGGA \\
\hline \multirow[t]{2}{*}{ U6 } & Forward: GCTTCGGCAGCACATATACTAAAAT \\
\hline & Reverse: CGCTTCACGAATTTGCGTGTCAT \\
\hline
\end{tabular}

miR, miRNA; LINC01232, long intergenic non-coding RNA LINC01232; HDGF, hepatoma-derived growth factor.

cultured medium supplemented with $10 \mu 1$ of CCK-8 solution (Dojindo Molecular Technologies, Inc.) was added to each well. Following incubation at $37^{\circ} \mathrm{C}$ for $2 \mathrm{~h}$, the absorbance was detected in each well at a wavelength of $450 \mathrm{~nm}$ using a microplate reader (BioRad Laboratories, Inc.).

Flow cytometry. Following $48 \mathrm{~h}$ of culture, the transfected cells were treated with ethylenediaminetetraacetic acid (EDTA)-free trypsin (Gibco; Thermo Fisher Scientific, Inc.) and washed with precooled phosphate-buffered saline (Gibco; Thermo Fisher Scientific, Inc.), followed by centrifugation at $4^{\circ} \mathrm{C}$ at $12,000 \mathrm{x}$ g for $5 \mathrm{~min}$. Subsequently, an Annexin V-fluorescein isothiocyanate (FITC) apoptosis detection kit (BioLegend, Inc.) was used to assess cell apoptosis quantitatively. The collected cells were suspended in $100 \mu \mathrm{l}$ of binding buffer and subsequently mixed with $10 \mu \mathrm{l}$ of Annexin V-FITC and $5 \mu \mathrm{l}$ of propidium iodide. Following $15 \mathrm{~min}$ of incubation at room temperature in the dark, the apoptotic rate was determined using a flow cytometer (FACScan; BD Biosciences).
Migration and invasion assays. For the invasion assays, the transfected cells were rinsed with phosphate-buffered saline and resuspended in FBS-free culture medium. Subsequently, $200 \mu \mathrm{l}$ of cell suspension containing $5 \times 10^{4}$ cells was plated into each upper compartment of a Biocoat Matrigel Invasion Chamber (BD Biosciences), while the lower compartments were loaded with $600 \mu \mathrm{l}$ of culture medium containing $20 \%$ FBS to serve as a chemoattractant. After $24 \mathrm{~h}$, the invaded cells were fixed with $4 \%$ paraformaldehyde at $37^{\circ} \mathrm{C}$ for $0.5 \mathrm{~h}$ and stained with $0.1 \%$ crystal violet at $37^{\circ} \mathrm{C}$ for $0.5 \mathrm{~h}$. The residual cells remaining on the upper surfaces were carefully wiped off with a cotton bud. For the migration assay, all experimental steps were performed as described above, except the chambers were not pre-coated with Matrigel. An inverted microscope (IX31; Olympus Corporation) was used to image the migrated and invaded cells. In total, 5 fields were randomly selected per insert, and the transmigrated cells were counted manually under a microscope as mentioned above. 
Xenograft mouse model. A short hairpin RNA (shRNA) targeting LINC01232 (sh-LINC01232) and scrambled shRNA (sh-ctrl) were designed and generated by GenePharma and inserted into the GenePharma Supersilencing Vector. Subsequently, the vectors were transfected into 293T cells (the Type Culture Collection of the Chinese Academy of Sciences) along with lentiviral packaging plasmids. The resulting lentiviruses were collected following $72 \mathrm{~h}$ of incubation at $37^{\circ} \mathrm{C}$, mixed with polybrene ( $5 \mu \mathrm{g} / \mathrm{ml}$; Sigma-Aldrich; Merck KGaA) and used to infect the Eca109 cells at an MOI of 5. Eca109 cells exhibiting stable sh-LINC01232 or sh-ctrl expression were selected using puromycin.

The animal experiments were conducted with the approval of the Institutional Animal Care and Use Committee of the Affiliated Hospital of Inner Mongolia University for the Nationalities. BALB/c nude mice $(n=6 ; 4-6$ weeks old) were purchased from Beijing Vital River Laboratory Animal Technology Co., Ltd., and kept in a specific pathogen-free environment at $25^{\circ} \mathrm{C}$ with $50 \%$ humidity, with a 10/14-h light/dark cycle and ad libitum food/water access. Mice were subcutaneously injected with Eca109 cells that stably expressed sh-LINC01232 or sh-ctrl. Each group included 3 mice. The sizes of the resulting subcutaneous xenografts were monitored by measuring the tumor length (L) and width (W) every 6 days. The tumor volume was calculated using the formula: Volume $=0.5 \times\left(\mathrm{L} \mathrm{x} \mathrm{W}^{2}\right)$. All mice were euthanized at the end of day 30 , and the tumor xenografts were imaged and weighed.

Bioinformatics analysis. GEPIA (http://gepia.cancer-pku.cn/) contained the TCGA and GTEx databases, and was used to analyze the LINC01232 expression profile in ESCC tumors $(n=182)$ and normal healthy tissues $(n=286)$. IncLocator (http://www.csbio.sjtu.edu.cn/bioinf/lncLocator/) was applied to predict the localization of LINC01232.

StarBase 3.0 (http://starbase.sysu.edu.cn/) was used to identify the potential miRNAs that may be adsorbed by LINC01232. The putative target of miR-654-3p was predicted by StarBase 3.0, TargetScan (http://www.targetscan.org/), and miRDB (http://mirdb.org/).

RNA immunoprecipitation (RIP) assay. To assess the interaction between LINC01232 and miR-654-3p in ESCC cells, RIP assay was performed using the Magna RIP RNA-Binding Protein Immunoprecipitation kit (EMD Millipore). ESCC cells were treated with RIP buffer. An anti-Argonaute2 (Ago2) antibody or normal mouse immunoglobulin $\mathrm{G}(\mathrm{IgG})$ antibody (1:5,000 dilution; both cat. no. 03-110; EMD Millipore) was conjugated to magnetic beads, which were then incubated overnight with whole-cell extracts at $4^{\circ} \mathrm{C}$. The magnetic beads were then harvested and treated with proteinase $\mathrm{K}$, and the immunoprecipitated RNA was extracted. The enrichment of LINC01232 and miR-654-3p in immunoprecipitated RNA was evaluated by RT-qPCR.

Luciferase reporter assay. The wild-type (wt) fragments of LINC01232 and HDGF 3'-UTR containing the miR-654-3p binding site were amplified by RT-qPCR and inserted downstream of the pmirGLO dual-luciferase vector (Promega Corporation) to yield the LINC01232-wt and HDGF-wt reporter plasmids, respectively. Binding site-directed mutagenesis was conducted using the GeneTailor ${ }^{\mathrm{TM}}$ Site-Directed
Mutagenesis System (Invitrogen; Thermo Fisher Scientific, Inc.) to generate LINC01232-mutant (LINC01232-mut) and HDGF-mut. ESCC cells were seeded in 24-well plates and co-transfected with miR-654-3p mimic or NC mimic and wt or mut reporter plasmids. Luciferase activity was measured $48 \mathrm{~h}$ following transfection using the Dual-Luciferase Reporter Assay System (Promega Corporation), and normalized to Renilla luciferase activity.

Western blot analysis. Total proteins were isolated from the cells using RIPA lysis buffer (Beyotime Institute of Biotechnology, Inc.), and the concentration in each lysate was measured using a BCA Protein Assay kit (Beyotime Institute of Biotechnology, Inc.). Equal amounts of total protein (30 $\mu \mathrm{g})$ were subjected to electrophoresis on $10 \%$ sodium dodecyl sulphate-polyacrylamide gel electrophoresis gels and transferred to polyvinylidene difluoride membranes. Membranes were blocked for $2 \mathrm{~h}$ in $5 \%$ non-fat milk at room temperature and then probed with primary antibodies overnight at $4^{\circ} \mathrm{C}$. Subsequently, the membranes were incubated with a horseradish peroxidase-conjugated goat anti-rabbit IgG secondary antibody (1:5,000 dilution; cat. no. ab205718; Abcam) at room temperature for $1 \mathrm{~h}$. Finally, Immobilon ${ }^{\circledR}$ ECL Ultra Western HRP Substrate (EMD Millipore) was used to visualize the signals corresponding to the labeled proteins. Primary antibodies specific for HDGF (cat. no. ab128921; Abcam) and GAPDH (cat. no. ab181603; Abcam) were used at a dilution of 1:1,000. Densitometry was performed using Quantity One software version 4.62 (Bio-Rad Laboratories, Inc.).

Statistical analysis. All data are presented as the means \pm standard deviation. Data from 2 groups were compared using a Student's t-test, whereas data from multiple groups were compared using one-way analysis of variance, followed by Tukey's test. Pearson's correlation coefficient was used to analyze the correlation among LINC01232, miR-654-3p and HDGF expression in ESCC tissues. The overall survival of ESCC patients was analyzed via the Kaplan-Meier method, and compared with the log-rank test. All statistical analyses were performed using SPSS version 19.0 software package (IBS SPSS, Inc.), and P-values $<0.05$ were considered to indicate statistically significant differences.

\section{Results}

Interference of LINC01232 inhibits the malignant characteristics of ESCC cells. Initially, GEPIA (http://gepia. cancer-pku.cn/) was used to analyze the LINC01232 expression profile in ESCC. Data from the TCGA and GTEx databases revealed high levels of LINC01232 expression in ESCC tissues compared to normal tissues (Fig. 1A). To confirm this observation, RT-qPCR was performed to determine the abundance of LINC01232 in 55 pairs of ESCC tissues and matched normal tissues. RT-qPCR validated the upregulation of LINC01232 in ESCC tissues compared with that in matched normal tissues (Fig. 1B). Additionally, RT-qPCR was used to determine the expression of LINC01232 in the ESCC cell lines, Eca109, TE-1 and KYSE150, with the HET-1A normal human esophageal epithelial cells used as the control. LINC01232 was 


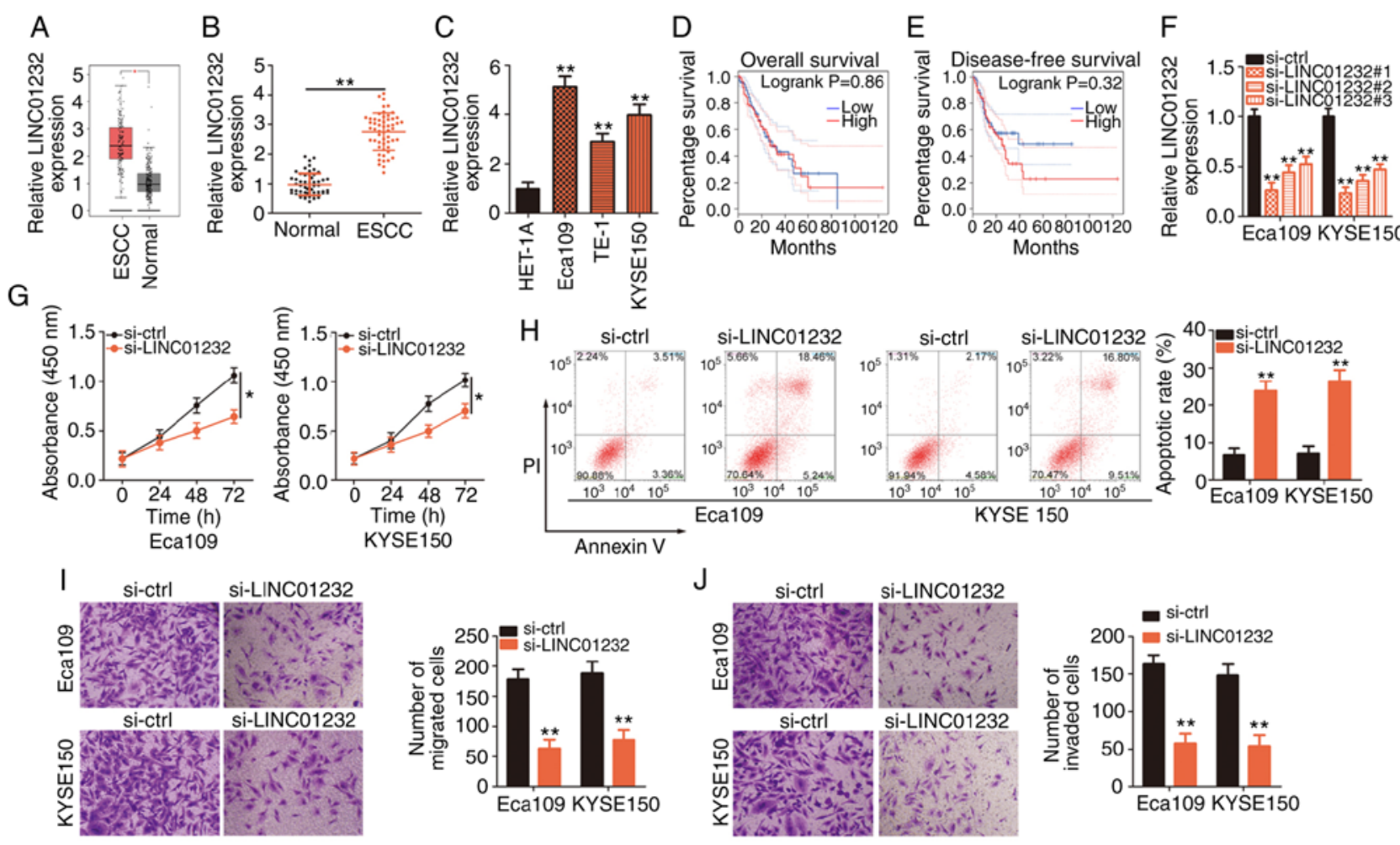

Figure 1. The downregulation of long intergenic non-coding RNA (LINC)01232 suppresses cell proliferation, migration and invasion, and promotes cell apoptosis in esophageal squamous cell carcinoma (ESCC). (A) GEPIA was used to analyze LINC01232 expression in ESCC tissues ( $\mathrm{n}=182$ ) and normal tissues ( $\mathrm{n}=286$ ). (B) LINC01232 expression in 55 pairs of ESCC tissues and matched normal tissues was detected by reverse transcription-quantitative polymerase chain reaction (RT-qPCR). (C) LINC01232 expression was detected in 3 ESCC cell lines (Eca109, TE-1 and KYSE150) and a normal human esophageal epithelial cell line (HET-1A) by RT-qPCR. (D and E) The association of LINC01232 expression with overall survival and disease-free survival in patients with ESCC were analyzed using GEPIA. (F) The silencing efficiency of si-LINC01232 against LINC01232 expression in Eca109 and KYSE150 cells was analyzed by RT-qPCR. (G and H) CCK-8 assay and flow cytometry were performed to measure the proliferation and apoptosis of Eca109 and KYSE150 cells subjected to LINC01232 depletion. (I and J) The migratory and invasive capabilities of Eca109 and KYSE150 cells following si-LINC01232 or si-ctrl transfection were evaluated using migration and invasion assays, respectively. ${ }^{*} \mathrm{P}<0.05$ and ${ }^{* *} \mathrm{P}<0.01$ vs. normal tissues or si-ctrl.

expressed at higher levels in all 3 ESCC cell lines compared with the control cells (Fig. 1C). The TCGA and GTEx data were analyzed to determine the clinical relevance of LINC01232 in ESCC and it was revealed that its expression was not associated with the overall survival (Fig. 1D) or disease-free survival (Fig. 1E) of patients with ESCC.

LINC01232 expression was most abundant in the Eca109 and KYSE150 cell lines; thus, these cell lines were selected for further experimental use. To determine the functional roles of LINC01232 in ESCC cellular processes, si-LINC01232 was designed to silence LINC01232 expression in the Eca109 and KYSE150 cells. The silencing efficiency was confirmed by RT-qPCR. Of the 3 siRNAs, si-LINC01232\#1 exhibited the most efficient interference and was thus used in subsequent experiments (Fig. 1F). Transfection with si-LINC01232 inhibited the proliferation (Fig. 1G) and promoted the apoptosis (Fig. 1H) of both the Eca109 and KYSE150 cells. Moreover, LINC01232 knockdown evidently impaired the migratory (Fig. 1I) and invasive capabilities (Fig. 1J) of the Eca109 and KYSE150 cells, as demonstrated by migration and invasion assays, respectively. In summary, LINC01232 expression was found to be upregulated in ESCC and to exert pro-oncogenic effects during cancer progression.

miR-654-3p is sponged by LINC01232 and serves as a tumor inhibitor in ESCC cells. To explore the mechanisms underlying the effects of LINC01232, the distribution of LINC01232 in ESCC cells was first analyzed. Using IncLocator (http://www.csbio.sjtu.edu.cn/bioinf/lncLocator/), it was determined that LINC01232 was distributed primarily in the cytoplasm (Fig. 2A), which was consistent with the results of cell cytosolic/nuclear fraction isolation and RT-qPCR analysis (Fig. 2B). Previous studies have revealed that cytoplasmic IncRNAs can serve as molecular sponges to prevent the suppression of target mRNAs by specific miRNAs (28-30). Therefore, it was hypothesize that LINC01232 may also serve as a sponge for miRNA and would thus regulate the expression of specific miRNAs.

A StarBase 3.0 search predicted 21 miRNAs as candidate targets of LINC01232 (Fig. 2C). Of these, miR-181a-5p (31), miR-21-5p (32) and miR-708-5p (33) have been found to be upregulated in ESCC, while miR-28-5p has been shown to exhibit a stable expression (34); therefore, these 4 miRNAs were excluded from subsequent analyses. Of the remaining candidates, miR-122-5p, miR-181b-5p, miR-181c-5p, miR-181d-5p, miR-211-5p, miR-370-5p, miR-493-5p, miR-506-5p, miR-590-5p, miR-642b-5p and miR-654-3p were selected, and their expression in LINC01232-deficient Eca109 and KYSE150 cells was evaluated. RT-qPCR analysis clearly affirmed the upregulation of miR-654-3p in Eca109 and KYSE150 cells following the silencing of LINC01232, whereas the expression of the other miRNAs remained 
A

IncLocator prediction result

\begin{tabular}{|l|l|}
\hline Subcellular locations & Score \\
\hline Cytoplasm & 0.490873951296 \\
\hline Nucleus & 0.307476206262 \\
\hline Ribosome & 0.0830770049645 \\
\hline Cytosol & 0.0826470623014 \\
\hline Exosome & 0.0359257751756 \\
\hline
\end{tabular}

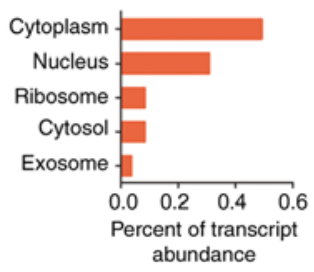

B

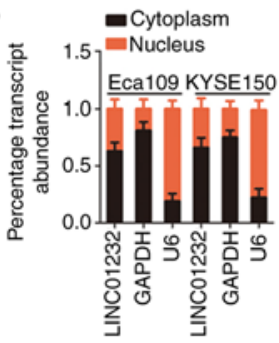

C

\begin{tabular}{|c|c|c|c|c|}
\hline miRNA name. & miRNA name. & miRNA name. & miRNA name & miRNA name \\
\hline miR-122-5p & miR-204-5p & miR-3127-5p & $\mathrm{miR}-4262$ & miR-590-5p \\
\hline miR-181a-5p & miR-211-5p & miR-3129. & miR-4761-3p. & $\operatorname{miR}-642 b-5 p$ \\
\hline$m i R-181 b-5 p$ & miR-21-5p & miR-3140-3p. & miR-493-5p & miR-654-3p. \\
\hline miR-181c-5p & miR-28-5p & miR-370-3p & miR-506-5p & miR-708-3p \\
\hline miR-181d-5p. & & & & \\
\hline
\end{tabular}

D

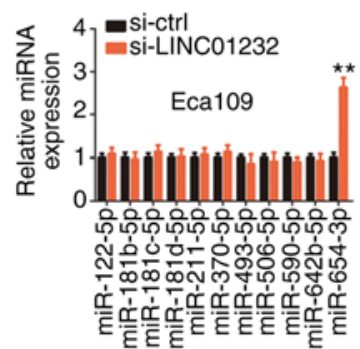

E

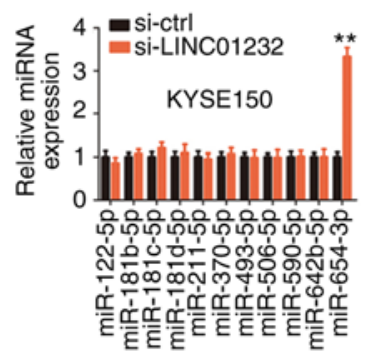

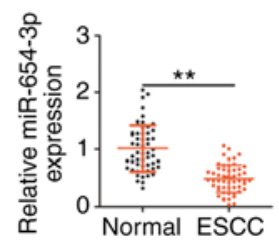

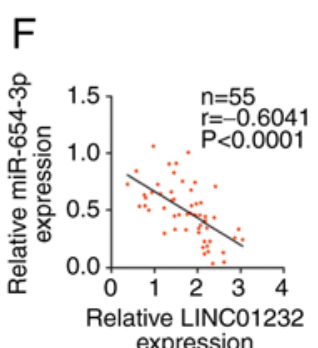

expression
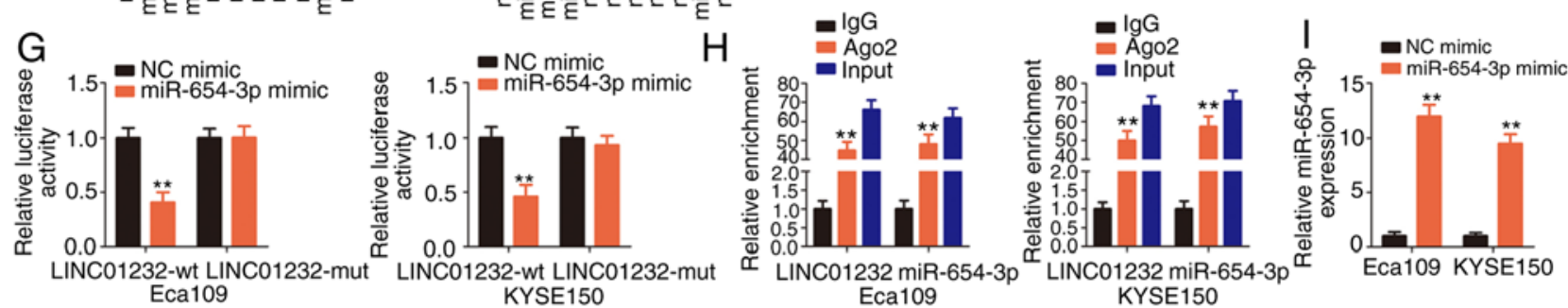

KYSE150
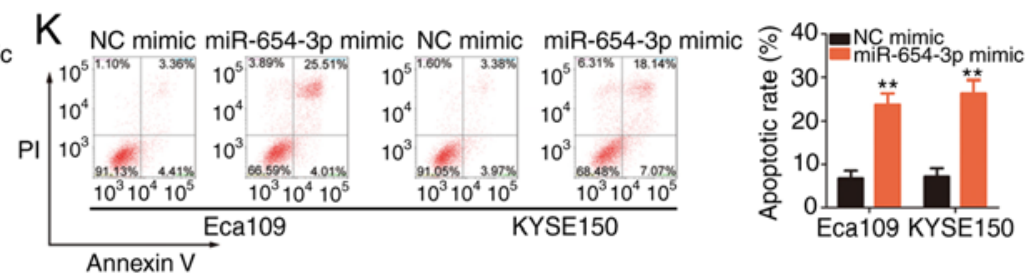

\section{$\mathrm{M}$}
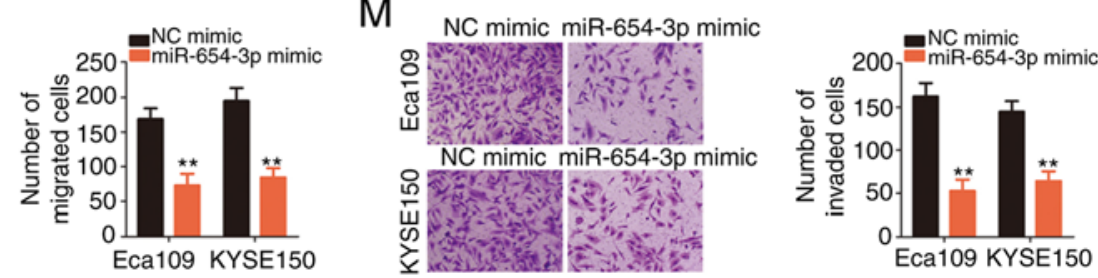

Figure 2. MicroRNA (miR)-654-3p is sponged by long intergenic non-coding RNA (LINC)01232 and plays an inhibitory role in esophageal squamous cell carcinoma (ESCC). (A) The lncLocator database was used to examine the subcellular location of LINC01232. (B) The subcellular location of LINC01232 was evaluated in cell cytosolic/nuclear fractions isolated from Eca109 and KYSE150 ESCC cells. (C) Potential LINC01232-interacting miRNAs identified through a StarBase 3.0 search. (D) The expression levels of miR-122-5p, miR-181b-5p, miR-181c-5p, miR-181d-5p, miR-211-5p, miR-370-5p, miR-493-5p, miR-506-5p, miR-590-5p, miR-642b-5p and miR-654-3p in LINC01232-deficient Eca109 and KYSE150 cells were detected by reverse transcription-quantitative polymerase chain reaction (RT-qPCR). (E) RT-qPCR was performed to detect miR-654-3p expression in 55 pairs of ESCC tissues and matched normal tissues. (F) Pearson's correlation coefficient illustrates the correlation between LINC01232 and miR-654-3p in 55 ESCC tissues. (G) Luciferase activity corresponding to LINC01232-wt or LINC01232-mut was detected in Eca109 and KYSE150 cells following transfection with the miR-654-3p mimic or NC mimic. (H) RT-qPCR was conducted to determine the enrichment of LINC01232 and miR-654-3p in Eca109 and KYSE150 cells after RNA immunoprecipitation. (I) Eca109 and KYSE150 cells were transfected with miR-654-3p mimic or NC mimic, and the overexpression efficiency was determined by RT-qPCR. (J-M) CCK-8 assay, flow cytometric analysis, and migration and invasion assays were performed to detect the proliferation, apoptosis, and migration and invasion of miR-654-3p-overexpressing Eca109 and KYSE150 cells. ${ }^{*} \mathrm{P}<0.05$ and ${ }^{* *} \mathrm{P}<0.01$ vs. NC mimic or si-ctrl. 

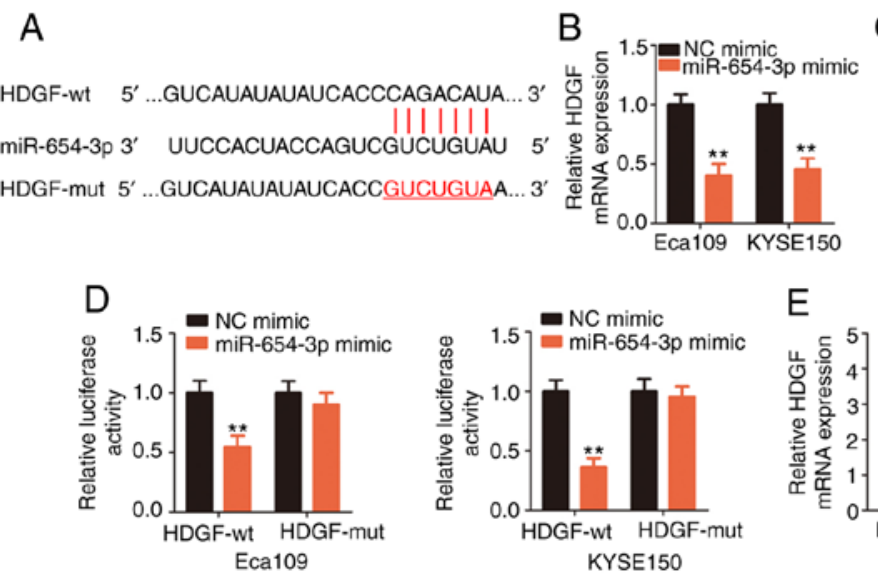

G

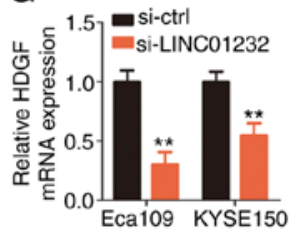

$J=\operatorname{si-ctrl}$

si-LINC01232+NC inhibitor - si-LINC01232+miR-654-3p inhibitor

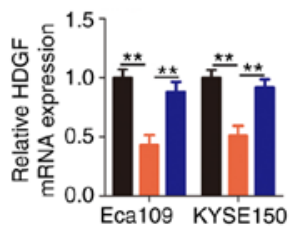

E

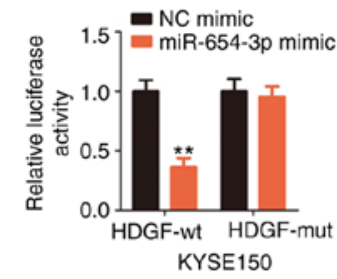

$\mathrm{H}$

K

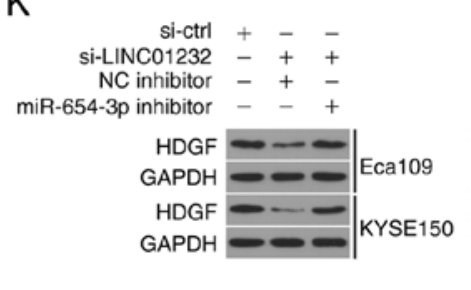

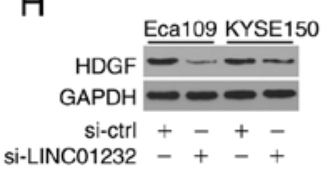

C

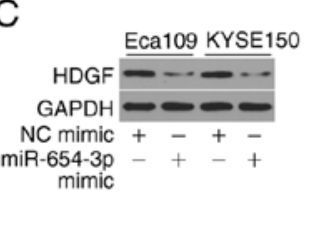

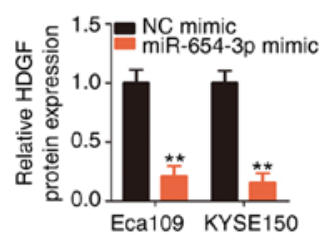

$\mathrm{F}$

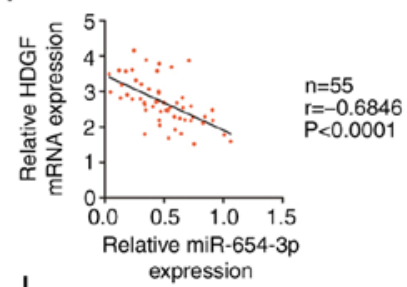

I
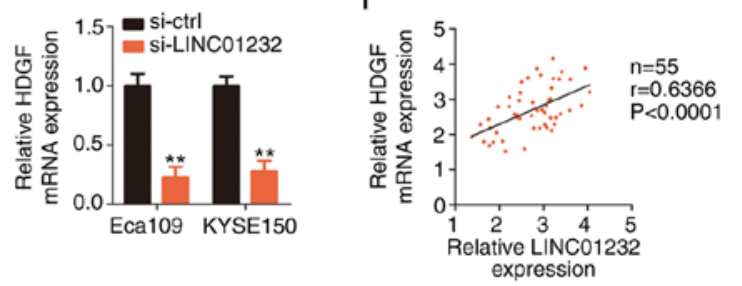

- si-ctrl

=si-LINC01232+NC inhibitor

- si-LINC01232+miR-654-3p inhibitor

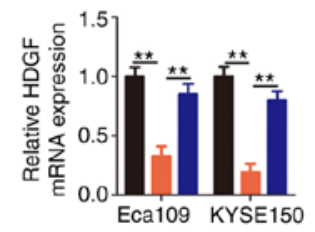

Figure 3. Long intergenic non-coding RNA (LINC)01232 positively regulates hepatoma-derived growth factor (HDGF) expression in esophageal squamous cell carcinoma (ESCC) cells by sequestering microRNA (miR)-654-3p. (A) Bioinformatics analyses were used to identify the complementary binding sites of miR-654-3p in the 3'-untranslated region of HDGF. The mutant binding sequences are also shown. (B and C) HDGF mRNA and protein levels were measured in Eca109 and KYSE150 cells following miR-654-3p overexpression by reverse transcription-quantitative polymerase chain reaction (RT-qPCR) and western blot analysis, respectively. (D) Wild-type (HDGF-wt) or mutant HDGF (HDGF-mut) reporter constructs were co-transfected into Eca109 and KYSE150 cells with miR-654-3p mimic or NC mimic. Luciferase activity was determined at $48 \mathrm{~h}$ following transfection. (E) RT-qPCR analysis detected HDGF mRNA expression in 55 pairs of ESCC tissues and matched normal tissues. (F) Pearson's correlation coefficient was used to determine the correlation between HDGF and miR-654-3p expression in 55 ESCC tissues. (G and H) RT-qPCR and western blot analysis were used to determine the regulatory effects of LINC01232 knockdown on HDGF mRNA and protein expression, respectively, in Eca109 and KYSE150 cells. (I) The correlation between LINC01232 and HDGF mRNA in the 55 ESCC tissues was determined by Pearson's correlation coefficient. (J and K) Small interfering RNA specific for LINC01232 (si-LINC01232) was co-transfected with miR-654-3p inhibitor or NC inhibitor into Eca109 and KYSE150 cells. Subsequent changes in the expression of HDGF mRNA and protein were determined by RT-qPCR and western blot analysis, respectively. ${ }^{* *} \mathrm{P}<0.01 \mathrm{vs.} \mathrm{NC} \mathrm{mimic} \mathrm{or} \mathrm{si-ctrl.}$

unaffected (Fig. 2D). Subsequently, miR-654-3p expression was detected in 55 pairs of ESCC tissues and matched normal tissues. The downregulation of miR-654-3p in ESCC tissues (Fig. 2E) indicated a negative correlation with LINC01232 expression (Fig. 2F; r $=-0.6041, \mathrm{P}<0.0001$ ). Additionally, luciferase reporter assay, which was performed to examine the binding between miR-654-3p and LINC01232 in ESCC cells, revealed a marked decrease in the luciferase activity of LINC01232-wt in response to miR-654-3p upregulation in the Eca109 and KYSE150 cells, whereas miR-654-3p overexpression had no effect on the luciferase activity associated with LINC01232-mut (Fig. 2G). Moreover, the considerable enrichment of LINC01232 and miR-654-3p was observed in $\mathrm{AGO} 2$-containing immunoprecipitation complexes when compared with IgG immunoprecipitates in RIP assay (Fig. 2H).

Subsequently, to assess the roles of miR-654-3p in ESCC cells, Eca109 and KYSE150 cells were transfected with miR-654-3p mimic or NC mimic and subjected to RT-qPCR analyses to detect miR-654-3p expression. Transfection with miR-654-3p mimic notably increased the expression of miR-654-3p in both the Eca109 and KYSE150 cells (Fig. 2I). The results of CCK- 8 assay revealed that the upregulation of miR-654-3p significantly hindered the proliferation of Eca109 and KYSE150 cells (Fig. 2J). The ectopic expression of miR-654-3p also markedly induced the apoptosis of the Eca109 and KYSE150 cells (Fig. 2K). Furthermore, miR-654-3p overexpression led to a marked decrease in the migration (Fig. 2L) and invasion (Fig. 2M) of the Eca109 and KYSE150 cells. In summary, these results indicated that miR-654-3p functioned as a cancer-inhibiting miRNA that was sponged by LINC01232 in ESCC cells.

LINC01232 functions as a ceRNA to positively regulate HDGF expression in ESCC cells by competitively binding to miR-654-3p. The TargetScan, miRDB and StarBase 3.0 databases were searched to identify the putative targets of miR-654-3p. HDGF (Fig. 3A) was selected for further evaluation given its important role in the oncogenicity of 

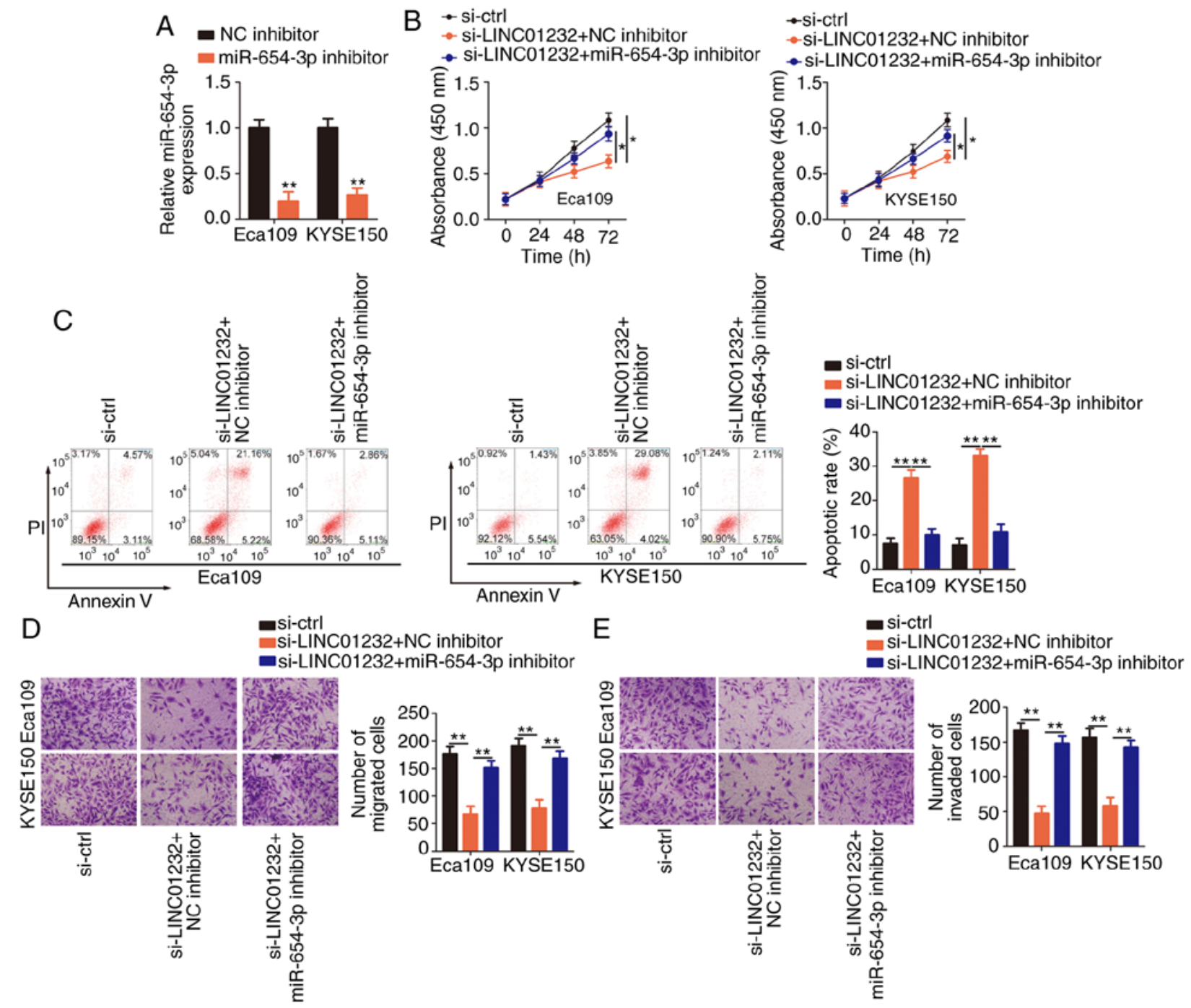

Figure 4. Inhibition of microRNA (miR)-654-3p abolishes the regulatory effects of long intergenic non-coding RNA (LINC)01232 knockdown in esophageal squamous cell carcinoma (ESCC) cells. (A) The expression of miR-654-3p in Eca109 and KYSE150 cells was detected by reverse transcription-quantitative polymerase chain reaction (RT-qPCR) following transfection with miR-654-3p inhibitor or NC inhibitor. (B and C) Eca109 and KYSE150 cells were co-transfected with small interfering RNA specific for LINC01232 (si-LINC01232) and miR-654-3p inhibitor or NC inhibitor. Cell proliferation and apoptosis were investigated using the CCK-8 assay and flow cytometry, respectively. (D and E) Migration and invasion assays were used to assess the migration and invasion capabilities, respectively of Eca109 and KYSE150 cells treated as described above. $\mathrm{P}<0.05$ and ${ }^{* * *} \mathrm{P}<0.01$ vs. NC inhibitor or sc-ctrl.

ESCC (35-37). In the present study, the results of RT-qPCR and western blot analyses demonstrated that the upregulation of miR-654-3p downregulated the expression of HDGF mRNA (Fig. 3B) and protein (Fig. 3C) in the Eca109 and KYSE150 cells. To further verify these findings, HDGF-wt and HDGF-mut reporter plasmids were generated and used in a luciferase reporter assay. Notably, miR-654-3p mimic markedly blocked the luciferase activity associated with HDGF-wt in the Eca109 and KYSE150 cells. However, the luciferase activity of HDGF-mut remained unaffected in response to miR-654-3p overexpression (Fig. 3D). Furthermore, a substantially upregulated HDGF mRNA expression was observed in ESCC tissues compared with matched normal tissues (Fig. 3E), and this expression negatively correlated with that of miR-654-3p (Fig. 3F; r=-0.6846, $\mathrm{P}<0.0001$ ).

To investigate the association among LINC01232, miR-654-3p and HDGF in ESCC cells, the HDGF mRNA and protein levels in LINC01232-deficient Eca109 and KYSE150 cells were evaluated by RT-qPCR and western blot analysis, respectively. The silencing of LINC01232 led to a significant decrease in HDGF expression at the mRNA (Fig. 3G) and protein level (Fig. 3H) in the Eca109 and KYSE150 cells. Notably, HDGF mRNA expression positively correlated with LINC01232 expression in ESCC tissues (Fig. 3I; $r=0.6366$, $\mathrm{P}<0.0001)$. Rescue experiments were also conducted using miR-654-3p inhibitor in Eca109 and KYSE150 cells subjected to LINC01232 silencing. LINC01232 knockdown induced decreases in the expression of both HDGF mRNA (Fig. 3J) and protein (Fig. 3K) in Eca109 and KYSE150 cells, which were restored by co-transfection with miR-654-3p inhibitor. These results indicate that LINC01232 positively regulates HDGF expression by competitively binding to miR-654-3p in ESCC cells.

LINC01232 functions by targeting the miR-654-3p/HDGF axis in ESCC cells. Rescue experiments were then performed to further verify the effects of the LINC01232/miR-654-3p/HDGF pathway on the malignant behaviors of ESCC cells. Briefly, 
A

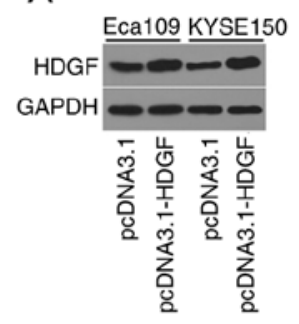

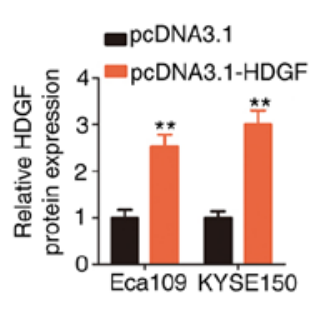

$\mathrm{B} \rightarrow$ si-ctrl

- si-LINC01232+pcDNA3.1

- si-LINC01232+pcDNA3.1-HDGF

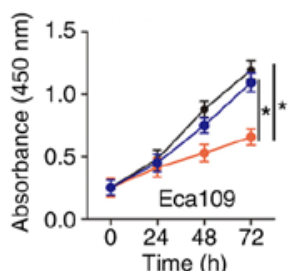

$\rightarrow$ si-ctrl

- si-LINC01232+pCDNA3.1

- si-LINC01232+pcDNA3.1-HDGF

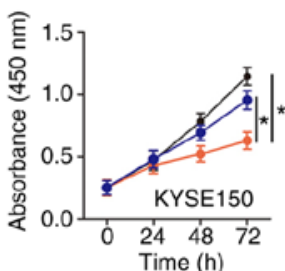

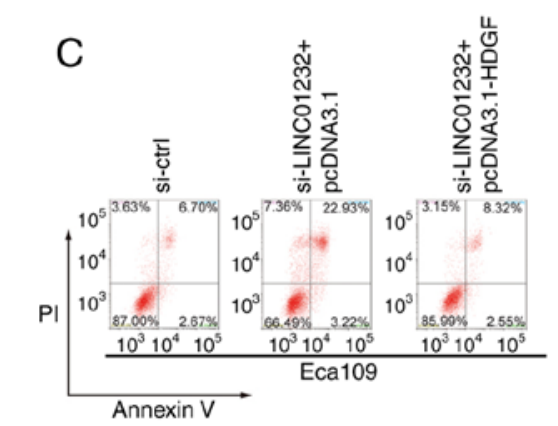

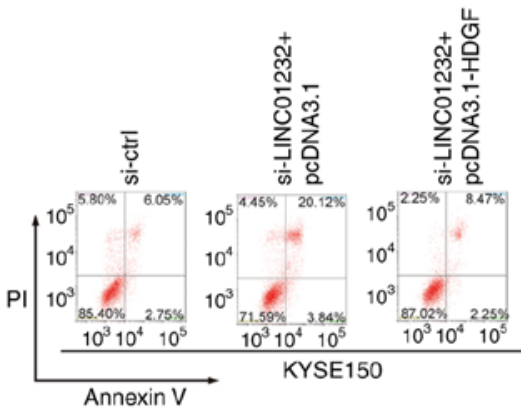

- si-ctrl

Si-LINC01232+pcDNA3.1

- si-LINC01232+pCDNA3.1-HDGF

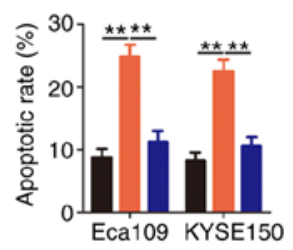

D

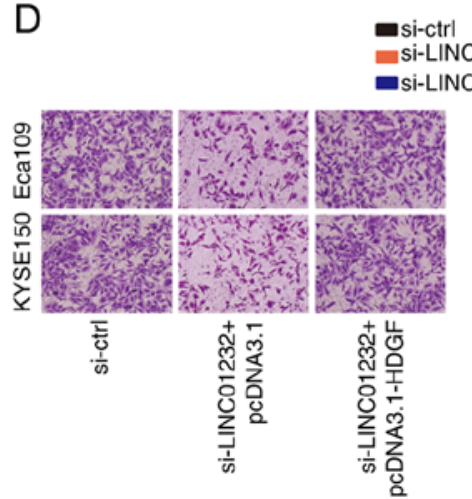

E

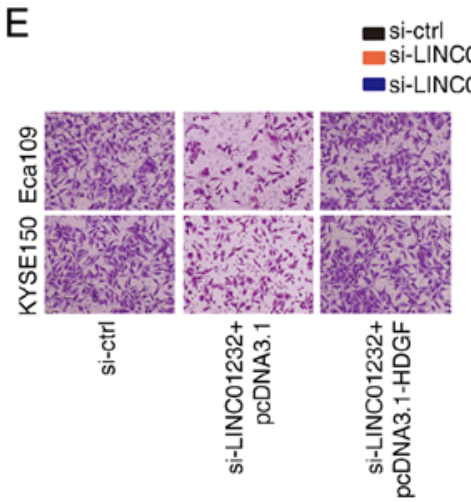

si-ctrl

1232+pcDNA3.1 $01232+$ pcDNA3.1-HDGF

Figure 5. Upregulation of hepatoma-derived growth factor (HDGF) reverses the effects of long intergenic non-coding RNA (LINC)01232 silencing in esophageal squamous cell carcinoma (ESCC) cells. (A) Western blot analysis was used to analyze the efficiency of pcDNA3.1-HDGF transfection in Eca109 and KYSE150 cells. (B-E) The pcDNA3.1-HDGF or pcDNA3.1 plasmid was introduced into Eca109 and KYSE150 cells in the presence of small interfering RNA specific for LINC01232 (si-LINC01232). Cell proliferation, apoptosis, and migration and invasion were assessed by CCK-8 assay, flow cytometry, and migration and invasion assays, respectively. ${ }^{*} \mathrm{P}<0.05$ and ${ }^{* *} \mathrm{P}<0.01$ vs. respective control.

si-LINC01232 and miR-654-3p inhibitor or NC inhibitor were co-transfected into the Eca109 and KYSE150 cells, which were then subjected to functional assays. The efficiency of miR-654-3p inhibitor in the Eca109 and KYSE150 cells was confirmed by RT-qPCR analysis (Fig. 4A). The inhibition of miR-654-3p was sufficient to abolish the changes in Eca109 and KYSE150 cell proliferation (Fig. 4B), apoptosis (Fig. 4C), migration (Fig. 4D) and invasion (Fig. 4E) induced by the silencing of LINC01232.

To examine whether HDGF is needed to achieve the effects of LINC01232 on the malignant phenotype of ESCC cells, either the HDGF-overexpressing plasmid pcDNA3.1-HDGF or the empty pcDNA3.1 vector were co-transfected with si-LINC01232 into the Eca109 and KYSE150 cells. The transfection efficiency of pcDNA3.1-HDGF was determined by western blot analysis (Fig. 5A). Indeed, the overexpression of HDGF partially reverse the impairment of Eca109 and KYSE150 cell proliferation induced by LINC01232 knockdown (Fig. 5B). Additionally, the apoptosis of Eca109 and KYSE150 cells induced by si-LINC01232 was partially abolished by co-transfection with pcDNA3.1-HDGF (Fig. 5C).
Furthermore, the upregulation of HDGF restored the migratory (Fig. 5D) and invasive capabilities (Fig. 5E) of the Eca109 and KYSE150 cells, which had been suppressed by LINC01232 silencing. Collectively, these results indicate that LINC01232 regulates ESCC cell activities at least partly by regulating the miR-654-3p/HDGF axis.

LINC01232 knockdown suppresses ESCC tumor growth in vivo. Finally, the effects of LINC01232 on ESCC tumor growth in vivo were examined using a xenograft mouse model. Eca109 cells were stably transfected with sh-LINC01232 or sh-ctrl and subcutaneously inoculated into nude mice. The subcutaneous xenografts grew at a markedly slower rate in the si-LINC01232 group than in the sh-ctrl group (Fig. 6A and B). The weights of the tumor xenografts were notably lower in the sh-LINC01232 group than in the sh-ctrl group (Fig. 6C). Following excision, the tumor xenografts were subjected to RT-qPCR and western blot analyses to determine the changes in the expression of LINC01232, miR-654-3p and HDGF. RT-qPCR analysis revealed the downregulation of LINC01232 (Fig. 6D) and the upregulation of miR-654-3p (Fig. 6E) in tumors originating 
A

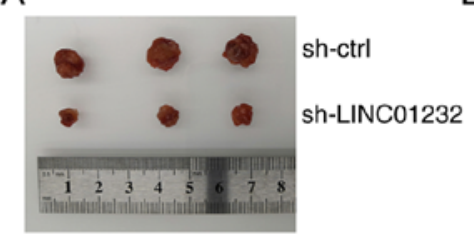

B

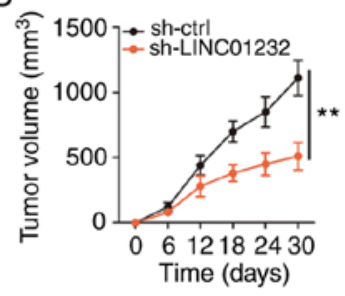

C

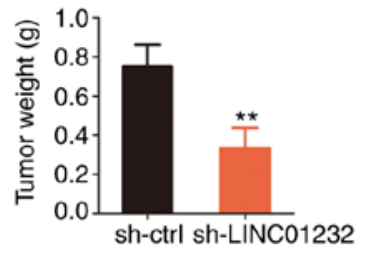

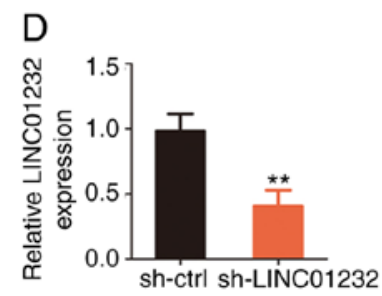

E
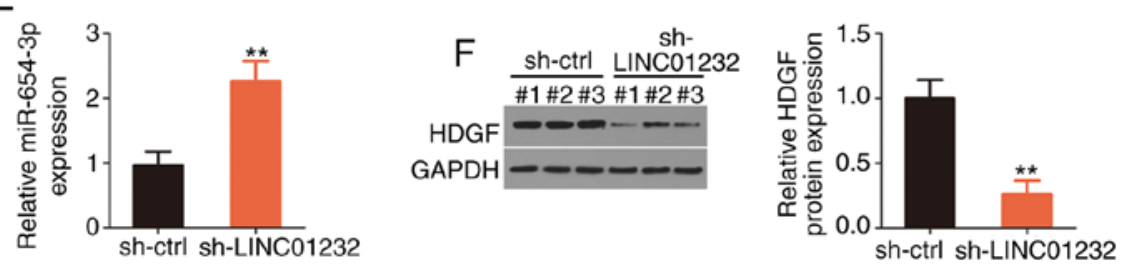

Figure 6. Loss of long intergenic non-coding RNA (LINC)01232 suppresses tumor growth in vivo. (A) Images of excised tumor xenografts. (B) Plots of tumor xenograft growth according to tumor volume, which was measured every 5 days. (C) Comparison of tumor weights obtained from nude mice euthanized at the end of day 30. (D and E) Analysis of LINC01232 and microRNA (miR)-654-3p expression in tumor xenografts was performed by reverse transcription-quantitative polymerase chain reaction. (F) HDGF protein expression was measured by western blot analysis in total protein isolated from tumor xenografts in the groups that were injected with ESCC cells treated with short hairpin RNA (sh) specific for LINC01232 or a negative control (sh-ctrl). ${ }^{* *} \mathrm{P}<0.01$ vs. sh-ctrl.

from the sh-LINC01232-transfected Eca109 cells. Western blot analysis also revealed the decreased HDGF protein level in the tumor xenografts collected from the sh-LINC01232 mice (Fig. 6F). On the whole, these results demonstrate the inhibitory effects of LINC01232 knockdown on ESCC tumor growth in vivo.

\section{Discussion}

Recent studies on IncRNAs have attracted increasing attention due to the involvement of these molecules in cancer oncogenesis and progression (38-40). Studies have reported the expression of hundreds of IncRNAs in ESCC, and differentially expressed lncRNAs have been shown to be closely related to ESCC malignancy through their effects on a wide range of tumor behaviors $(41,42)$. Therefore, it is necessary to clarify the functions of lncRNAs in ESCC and thus identify effective diagnostic markers and treatment targets. In the present study, the regulatory functions of LINC01232 in ESCC cells were examined and the mechanisms through which this molecule affects the malignant characteristics of this tumor type were investigated. Using both in vitro and in vivo functional experiments, the pro-oncogenic functions of LINC01232 and its interactions with miR-654-3p and HDGF in ESCC were examined.

LINC01232 expression is upregulated in pancreatic adenocarcinoma, and a high level of expression is associated with a poor clinical outcome (26). LINC01232 functions as an oncogenic lncRNA in pancreatic adenocarcinoma and regulates cell proliferation, apoptosis, migration, invasion and the epithelial-mesenchymal transition (26). However, the expression and roles of LINC01232 in ESCC have not yet been elucidated.

Herein, expression pattern analyses of lncRNAs in ESCC were conducted using the TCGA and GTEx databases. LINC01232 was identified as one of the most dysregulated IncRNAs in ESCC. Subsequently, RT-qPCR was performed to measure the expression of LINC01232 in ESCC tissues and cell lines. LINC01232 was highly expressed in ESCC tissues and cell lines, suggesting that this molecule plays crucial roles in ESCC progression. However, the expression high of LINC01232 was not found to be associated with the overall survival or disease-free survival of patients with ESCC. This finding suggests that LINC01232 has no potential to be investigated as a prognostic marker for ESCC. Through a series of functional in vitro experiments, it was determined that LINC01232 played carcinogenic roles in ESCC and that LINC01232 interference suppressed cell proliferation, migration, and invasion and promoted cell apoptosis. Moreover, a xenograft mouse model was established and the cancer-promoting roles of LINC01232 in ESCC tumor growth were examined in vivo.

In recent years, studies have increasingly demonstrated that IncRNAs function as ceRNAs that may sponge miRNAs to decrease their expression and activity, which would thereby regulate the suppressive effects on miRNA targets (43-45). In the present study, IncLocator analysis revealed that LINC01232 was mostly localized in the cytoplasm, consistent with the results of the cell cytosolic/nuclear fraction isolation experiment. This observation suggests that LINC01232 interacts with miRNAs in the cytoplasm. Initially, the target prediction algorithm indicated a potential interaction between LINC01232 and miR-654-3p. Herein, RT-qPCR was first used to confirm that LINC01232 silencing led to an increased miR-654-3p expression in ESCC cells. Additionally, it was observed that miR-654-3p and LINC01232 exhibited a negative correlation in ESCC tissues. Furthermore, luciferase reporter assay revealed that miR-654-3p could bind directly to the 'seed site' of LINC01232 in ESCC cells. RIP assays revealed that both LINC01232 and miR-654-3p were enriched in AGO2-containing immunoprecipitation complexes in ESCC cells. Furthermore, LINC01232 controlled the expression of HDGF, a direct target of miR-654-3p, by sponging miR-654-3p in ESCC cells. Accordingly, the results of the present study indicate a ceRNA process that involves LINC01232, miR-654-3p and HDGF in ESCC cells.

miR-654-3p is aberrantly expressed in several types of human cancers $(46,47)$. However, its expression status and 
roles in ESCC remain to be elucidated. In the present study, a weak miR-654-3p expression was observed in ESCC and it was determined that its upregulation suppressed the oncogenicity of ESCC cells. Subsequent mechanistic analyses validated HDGF as a downstream target of miR-654-3p in ESCC cells. HDGF, which is encoded by a gene located on the q21-q23 region of chromosome 1, is highly expressed in ESCC (36). Patients with ESCC having a high HDGF expression manifest a poorer disease-free and overall survival compared with patients with a low HDGF expression (36). Furthermore, HDGF is identified as an independent prognostic factor for ESCC patients with early stage (36). Functionally, HDGF exerts cancer-promoting roles, and contributes to tumor progression through its effects on various aggressive processes (35-37). The results of the rescue experiments reconfirmed that the effects of LINC01232 deficiency on ESCC cells could be reversed by increasing the output of the miR-654-3p/HDGF axis. The findings indicate that LINC01232 competitively binds to and acts as a molecular sponge for miR-654-3p, which in turn activates HDGF and affects ESCC malignancy.

LINC01232 is upregulated in ESCC and plays a tumor-promoting role during cancer progression by sequestering miR-654-3p to increase HDGF expression. Accordingly, the LINC01232/miR-654-3p/HDGF pathway may provide a novel theoretical basis for the management of ESCC.

\section{Acknowledgements}

Not applicable.

\section{Funding}

No funding was received.

\section{Availability of data and materials}

The datasets used and/or analyzed during the present study are available from the corresponding author on reasonable request.

\section{Authors' contributions}

MZ and HC designed the study. All authors (MZ, HC, BZ, ML and HM) performed the functional experiments. $\mathrm{MZ}$ and HC wrote the manuscript. All authors reviewed and edited the manuscript. All authors read and approved the manuscript and agree to be accountable for all aspects of the research in ensuring that the accuracy or integrity of any part of the work are appropriately investigated and resolved.

\section{Ethics approval and consent to participate}

Human clinical tissues were collected and used in accordance with the Institutional Ethics Committee of the Affiliated Hospital of Inner Mongolia University for the Nationalities. Additionally, written informed consent was provided by all patients before tissue collection. The animal experiments were conducted with approval from the Institutional Animal Care and Use Committee of Affiliated Hospital of Inner Mongolia University for the Nationalities.

\section{Patient consent for publication}

Not applicable.

\section{Competing interests}

The authors declare that they have no competing interests.

\section{References}

1. Bray F, Ferlay J, Soerjomataram I, Siegel RL, Torre LA and Jemal A: Global cancer statistics 2018: GLOBOCAN estimates of incidence and mortality worldwide for 36 cancers in 185 countries. CA Cancer J Clin 68: 394-424, 2018.

2. Torre LA, Bray F, Siegel RL, Ferlay J,Lortet-Tieulent J and Jemal A: Global cancer statistics, 2012. CA Cancer J Clin 65: 87-108, 2015.

3. Funakawa K, Uto H, Sasaki F, Nasu Y, Mawatari S, Arima S, Nakazawa J, Taguchi H, Hashimoto S, Kanmura S, et al: Effect of endoscopic submucosal dissection for superficial esophageal neoplasms and risk factors for postoperative stricture. Medicine (Baltimore) 94: e373, 2015.

4. Smyth EC, Lagergren J, Fitzgerald RC, Lordick F, Shah MA, Lagergren P and Cunningham D: Oesophageal cancer. Nat Rev Dis Primers 3: 17048, 2017.

5. Enzinger PC and Mayer RJ: Esophageal cancer. N Engl J Med 349: 2241-2252, 2003.

6. Zhang Y: Epidemiology of esophageal cancer. World J Gastroenterol 19: 5598-5606, 2013

7. Song Y, Li L, Ou Y, Gao Z, Li E, Li X, Zhang W, Wang J, Xu L, Zhou Y, et al: Identification of genomic alterations in oesophageal squamous cell cancer. Nature 509: 91-95, 2014.

8. Bach DH and Lee SK: Long noncoding RNAs in cancer cells. Cancer Lett 419: 152-166, 2018.

9. Cao W, Liu JN, Liu Z, Wang X, Han ZG, Ji T, Chen WT and Zou X: A three-lncRNA signature derived from the Atlas of ncRNA in cancer (TANRIC) database predicts the survival of patients with head and neck squamous cell carcinoma. Oral Oncol 65: 94-101, 2017.

10. Sarfi M, Abbastabar M and Khalili E: Long noncoding RNAs biomarker-based cancer assessment. J Cell Physiol 234: 16971-16986, 2019.

11. Sun Y and Ma L: New insights into long non-coding RNA MALAT1 in cancer and metastasis. Cancers (Basel) 11: 216, 2019.

12. Ponting CP, Oliver PL and Reik W: Evolution and functions of long noncoding RNAs. Cell 136: 629-641, 2009.

13. Javed Z, Khan K, Sadia H, Raza S, Salehi B, Sharifi-Rad J and Cho WC: LncRNA and Wnt signaling in colorectal cancer. Cancer Cell Int 20: 326, 2020.

14. Tang WW, Wu Q, Li SQ, Tong YS, Liu ZH, Yang TX, Xu Y and Cao XF: Implication of lncRNAs in pathogenesis of esophageal cancer. Onco Targets Ther 8: 3219-3226, 2015.

15. Luo F, Wen Y, Zhou H and Li Z: Roles of long non-coding RNAs in cervical cancer. Life Sci 256: 117981, 2020.

16. Du F, Guo T and Cao C: Restoration of UPK1A-AS1 expression suppresses cell proliferation, migration, and invasion in esophageal squamous cell carcinoma cells partially by sponging microRNA-1248. Cancer Manag Res 12: 2653-2662, 2020.

17. Ren P, Zhang H, Chang L, Hong XD and Xing L: LncRNA NR2F1-AS1 promotes proliferation and metastasis of ESCC cells via regulating EMT. Eur Rev Med Pharmacol Sci 24: 3686-3693, 2020.

18. Liang $X, W u ~ Z$, Shen S, Niu Y, Guo Y, Liang J and Guo W: LINC01980 facilitates esophageal squamous cell carcinoma progression via regulation of miR-190a-5p/MYO5A pathway. Arch Biochem Biophys 686: 108371, 2020.

19. Bartel DP: MicroRNAs: Target recognition and regulatory functions. Cell 136: 215-233, 2009.

20. Shukla GC, Singh J and Barik S: MicroRNAs: Processing, maturation, target recognition and regulatory functions. Mol Cell Pharmacol 3: 83-92, 2011.

21. Sharma P and Sharma R: miRNA-mRNA crosstalk in esophageal cancer: From diagnosis to therapy. Crit Rev Oncol Hematol 96: 449-462, 2015.

22. Du G, Zhou J, Cheng L, Ma X, Gui Y and Tan B: High expression of miR-206 predicts adverse outcomes: A potential therapeutic target for esophageal cancer. Comb Chem High Throughput Screen 22: 599-611, 2019. 
23. Wang W, Fu S, Lin X, Zheng J, Pu J, Gu Y, Deng W, Liu Y, He Z, Liang $\mathrm{W}$ and Wang C: miR-92b-3p functions as a key gene in esophageal squamous cell cancer as determined by co-expression analysis. Onco Targets Ther 12: 8339-8353, 2019.

24. Hu X, Wang M, Cao L, Cong L, Gao Y, Lu J, Feng J, Shen B and Liu D: miR-4319 suppresses the growth of esophageal squamous cell carcinoma via targeting NLRC5. Curr Mol Pharmacol 13: 144-149, 2020.

25. Ye Y, Shen A and Liu A: Long non-coding RNA H19 and cancer: A competing endogenous RNA. Bull Cancer 106: 1152-1159, 2019.

26. Li Q, Lei C, Lu C, Wang J, Gao M and Gao W: LINC01232 exerts oncogenic activities in pancreatic adenocarcinoma via regulation of TM9SF2. Cell Death Dis 10: 698, 2019.

27. Livak KJ and Schmittgen TD: Analysis of relative gene expression data using real-time quantitative PCR and the 2(-Delta Delta C(T)) method. Methods 25: 402-408, 2001

28. Bayoumi AS, Sayed A, Broskova Z, Teoh JP, Wilson J, Su H, Tang YL and Kim IM: Crosstalk between long noncoding RNAs and microRNAs in health and disease. Int J Mol Sci 17: 356, 2016

29. Abdollahzadeh R, Daraei A, Mansoori Y, Sepahvand M, Amoli MM and Tavakkoly-Bazzaz J: Competing endogenous RNA (ceRNA) cross talk and language in ceRNA regulatory networks: A new look at hallmarks of breast cancer. J Cell Physiol 234: 10080-10100, 2019.

30. Dong BS, Shi MJ, Su SB and Zhang H: Insight into long noncoding competing endogenous RNA networks in hepatic fibrosis: The potential implications for mechanism and therapy. Gene 687: 255-260, 2019.

31. Xiang Z, Dong X, Sun Q, Li X and Yan B: Clinical significance of up-regulated miR-181a in prognosis and progression of esophageal cancer. Acta Biochim Biophys Sin (Shanghai) 46 1007-1010, 2014

32. Xia Y, Wang Y, Wang Q, Ghaffar M, Wang Y, Sheng W and Zhang F: Increased miR-203-3p and reduced miR-21-5p synergistically inhibit proliferation, migration, and invasion in esophageal cancer cells. Anticancer Drugs 30: 38-45, 2019.

33. Yang H, Su H, Hu N, Wang C, Wang L, Giffen C, Goldstein AM, Lee MP and Taylor PR: Integrated analysis of genome-wide miRNAs and targeted gene expression in esophageal squamous cell carcinoma (ESCC) and relation to prognosis. BMC Cancer 20: 388, 2020.

34. Chen L, Jin Y, Wang L, Sun F, Yang X, Shi M, Zhan C, Shi Y and Wang Q: Identification of reference genes and miRNAs for qRT-PCR in human esophageal squamous cell carcinoma. Med Oncol 34: 2, 2017.

35. Matsuyama A, Inoue H, Shibuta K, Tanaka Y, Barnard GF, Sugimachi K and Mori M: Hepatoma-derived growth factor is associated with reduced sensitivity to irradiation in esophageal cancer. Cancer Res 61: 5714-5717, 2001.
36. Yamamoto S, Tomita Y, Hoshida Y, Morii E, Yasuda T, Doki Y, Aozasa K, Uyama H, Nakamura H and Monden M: Expression level of hepatoma-derived growth factor correlates with tumor recurrence of esophageal carcinoma. Ann Surg Oncol 14: 2141-2149, 2007.

37. Bao $\mathrm{CH}$, Wang XT, Ma W, Wang NN, Nesa EU, Wang JB, Wang C, Jia YB, Wang K, Tian $\mathrm{H}$ and Cheng YF: Irradiated fibroblasts promote epithelial-mesenchymal transition and HDGF expression of esophageal squamous cell carcinoma. Biochem Biophys Res Commun 458: 441-447, 2015.

38. Huang Z, Zhou JK, Peng Y, He W and Huang C: The role of long noncoding RNAs in hepatocellular carcinoma. Mol Cancer 19: 77, 2020.

39. Mozdarani H, Ezzatizadeh V and Rahbar Parvaneh R: The emerging role of the long non-coding RNA HOTAIR in breast cancer development and treatment. J Transl Med 18: 152, 2020.

40. Khajehdehi M, Khalaj-Kondori M, Ghasemi T, Jahanghiri B and Damaghi M: Long noncoding RNAs in gastrointestinal cancer: Tumor suppression versus tumor promotion. Dig Dis Sci Mar 17, 2020 (Epub ahead of print). doi: 10.1007/s10620-020-06200-x.

41. Feng Q, Zhang H, Yao D, Chen WD and Wang YD: Emerging role of non-coding RNAs in esophageal squamous cell carcinoma. Int J Mol Sci 21: 258, 2019.

42. Talebi A, Masoodi M, Mirzaei A, Mehrad-Majd H, Azizpour M and Akbari A: Biological and clinical relevance of metastasis-associated long noncoding RNAs in esophageal squamous cell carcinoma: A systematic review. J Cell Physiol 235: 848-868, 2020.

43. Wang JJ, Huang YQ, Song W, Li YF, Wang H, Wang WJ and Huang M: Comprehensive analysis of the IncRNA associated competing endogenous RNA network in breast cancer. Oncol Rep 42: 2572-2582, 2019.

44. Pan H, Guo C, Pan J, Guo D, Song S, Zhou Y and Xu D: Construction of a competitive endogenous RNA network and identification of potential regulatory axis in gastric cancer. Front Oncol 9: 912, 2019.

45. Liang W and Sun F: Identification of pivotal lncRNAs in papillary thyroid cancer using lncRNA-mRNA-miRNA ceRNA network analysis. PeerJ 7: e7441, 2019.

46. Yang J, Zhang Z, Chen S, Dou W, Xie R and Gao J: miR-654-3p predicts the prognosis of hepatocellular carcinoma and inhibits the proliferation, migration, and invasion of cancer cells. Cancer Biomark 28: 73-79, 2020.

47. Li P, Cai JX, Han F, Wang J, Zhou JJ, Shen KW and Wang LH: Expression and significance of miR-654-5p and miR-376b-3p in patients with colon cancer. World J Gastrointest Oncol 12: $492-502,2020$

(i) $($ This work is licensed under a Creative Commons Attribution-NonCommercial-NoDerivatives 4.0 International (CC BY-NC-ND 4.0) License. 